\title{
Successful Treatment of Vasculo-Behcet's Disease Presenting as Recurrent Pseudoaneurysms: the Importance of Medical Treatment
}

\author{
Shuang Li $\cdot$ Ai-Jun Chen $\cdot$ Kun Huang $\cdot$ Hui Li
}

To view enhanced content go to www.dermtherapy-open.com

Received: March 24, 2013 / Published online: May 14, 2013

(C) The Author(s) 2013. This article is published with open access at Springerlink.com

\section{ABSTRACT}

Introduction: Vasculo-Behcet's disease is a subtype of Behcet's disease, characterized by cases in which vascular complications are present and often dominate the clinical features. In this disease, there are four different vascular complications: arterial occlusion, arterial aneurysm or pseudoaneurysm, venous thrombosis, and variceal formation. It is rare that arterial lesions are multiple, but without venous involvement. So far, the optimal treatment of the disease has not been established.

Case Report: The authors report a rare case of vasculo-Behcet's disease with multiple and

S. Li and A.-J. Chen contributed equally to this work.

S. Li · A.-J. Chen · K. Huang (ه) · H. Li

The Department of Dermatology, The First Affiliated

Hospital, Chongqing Medical University, No. 1

Youyi Road, Chongqing 400016, China

e-mail: feelingkun@126.com recurrent pseudoaneurysms in large arteries, but without affecting the venous system. The patient underwent three rounds of surgery, but developed a new pseudoaneurysm after each operation in short term. However, the patient was successfully treated with a combination of prednisone and immunosuppressive agents.

Conclusion: For Vasculo-Behcet's disease, surgical and endovascular interventions alone increased the incidence of pseudoaneurysm. Early diagnosis and early initiation of prednisone in combination with immunosuppressive therapy are critical for inhibiting the progression of vascular lesions and provide a good prognosis.

Keywords: Azathioprine; Behcet's disease; Immunosuppressive agents; Intravenous pulse cyclophosphamide;

Prednisone; Pseudoaneurysms; Surgical therapy; VasculoBehcet's disease

\section{INTRODUCTION}

Behcet's disease is a systemic vasculitis disorder of unknown etiology, characterized by relapsing episodes of oral aphthous ulcers, genital ulcers, 
ocular lesions, and skin lesions. It can affect other systems, including vascular, gastrointestinal, and neurological systems [1]. The concept of vasculo-Behcet's disease has been adopted for cases in which vascular complications are present and often dominate the clinical features [2]. It is not uncommon in Behcet's disease, with a prevalence of $12.8 \%$ in China [3] and $14.3 \%$ in Turkey [4]. However, only $3.6 \%$ of patients with Behcet's disease have symptoms that affect the large arteries [4]. In most reports, arterial lesions are isolated and frequently coexist with venous lesions; rarely, there can be multiple lesions [4]. To the authors' knowledge, the treatment of the disease is difficult. In this paper, the authors reported a rare case of vasculo-Behcet's disease with multiple and recurrent pseudoaneurysms in large arteries, but without affecting the venous system. Surgical therapy alone increased the incidence of pseudoaneurysm. However, the patient was successfully treated with a combination of prednisone and immunosuppressive agents instead of surgery.

\section{METHODS}

All procedures followed were in accordance with the ethical standards of the responsible committee on human experimentation (institutional and national) and with the Helsinki Declaration of 1975, revised in 2000. Informed consent was obtained from the patient included in this study.

\section{CASE REPORT}

A 33-year-old Chinese male was admitted to the surgical ward due to a pulsating subcutaneous mass in the right subclavicular region over a 2-month period. The patient denied any history of trauma or manipulation. The patient felt progressive and intermittent numbness of the right upper extremity caused by the rapidly growing mass. A computed tomography (CT) angiogram revealed a right subclavian artery pseudoaneurysm of $4.5 \mathrm{~cm} \times 3.5 \mathrm{~cm}$ (Fig. 1a), which was soon resected and an expanded polytetrafluoroethylene (e-PTFE) vascular graft was performed. Histology of the arterial wall revealed inflammatory cell infiltrate (Fig. 2).

Two months later, the patient developed a symptomatic recurrence at the surgical site. A CT angiography revealed that there were both proximal and distal anastomotic pseudoaneurysms, $\quad 0.76 \mathrm{~cm} \times 0.87 \mathrm{~cm}$ and $4.45 \mathrm{~cm} \times 4.67 \mathrm{~cm}$, respectively, at the right subclavian artery (Fig. 1b). The two anastomotic pseudoaneurysms were also excised.

Four months after the second operation, the patient suffered from persistent severe left lumbago that responded poorly to analgesics. An abdominal CT angiography revealed aneurysmal dilatation of the upper abdominal aorta and the celiac trunk (Fig. 1c). Moreover, the left kidney and adrenal gland were atrophied due to compression by aneurysmal dilatation. Consequently, the patient underwent another operation.

One month after the third operation, the patient had a pseudoaneurysm of $2.35 \mathrm{~cm} \times 1.74 \mathrm{~cm}$ in the right thoracoacromial artery (Fig. 1d). Because the patient developed multiple and recurrent aneurysms over a short time span, the patient was admitted to the dermatological ward instead of reoperation.

After admission, a detailed review of the patient's history revealed recurrent oral and genital aphthous ulcers of 10-year duration. The ulcers occurred at least eight times in a year and were described as multiple painful lesions, each 

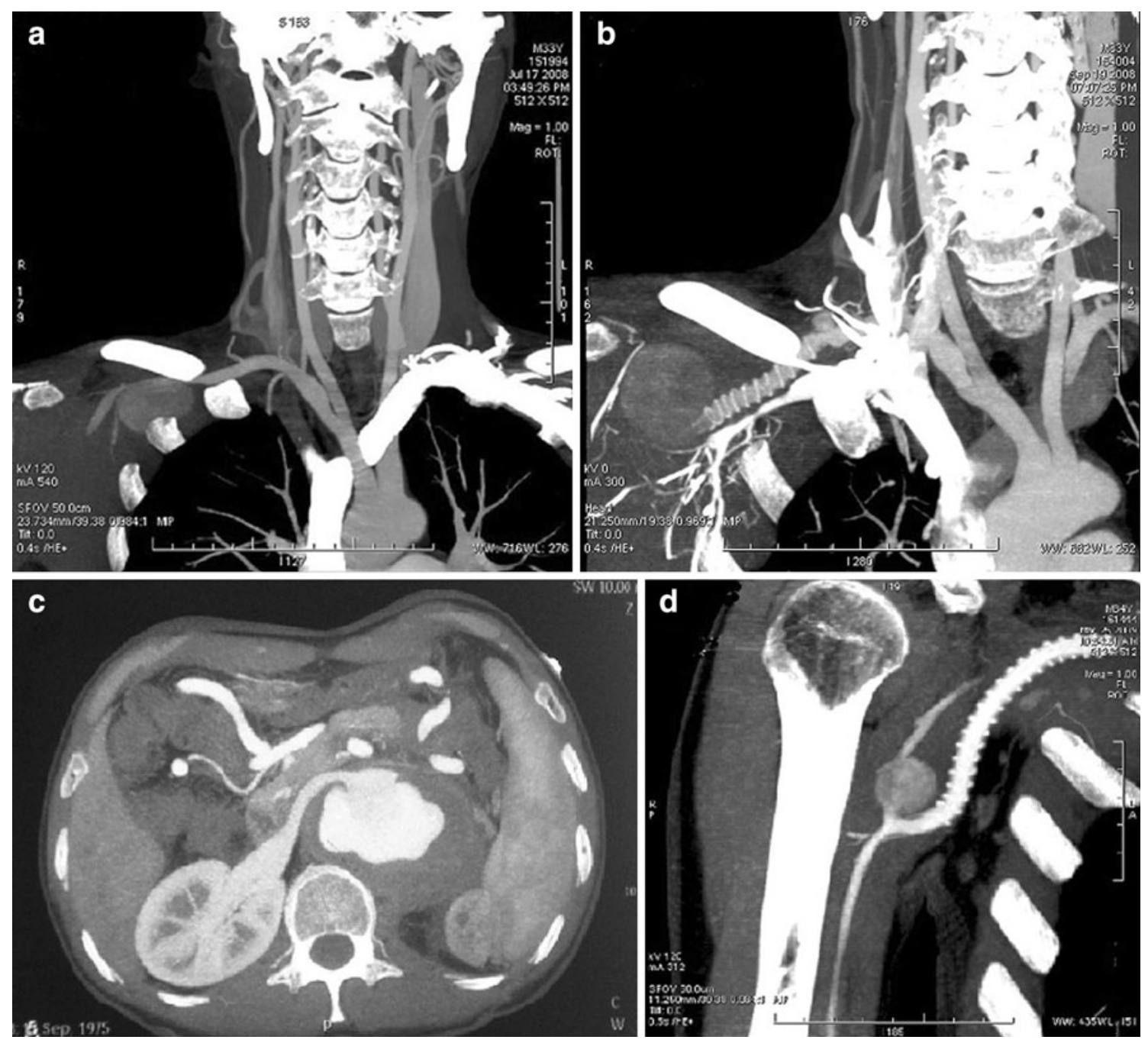

Fig. 1 Computed tomography angiography showing a right subclavian artery pseudoaneurysm of $4.5 \mathrm{~cm} \times 3.5 \mathrm{~cm}(\mathbf{a})$; the proximal and distal anastomotic pseudoaneurysms, $0.76 \mathrm{~cm} \times 0.87 \mathrm{~cm}$ and $4.45 \mathrm{~cm} \times 4.67 \mathrm{~cm}$, respectively, at

of which had a diameter of $2-5 \mathrm{~mm}$. The patient also had a history of recurrent pseudofolliculitis on the neck and back for 7 years. Physical examinations showed a pulsatile swelling in the right axilla. Multiple pigmented macules were seen on the neck and back (Fig. 3). The pathergy test was positive. No oral or genital lesions were detected. Neither eye disease nor joint problems were evident. Laboratory investigation revealed erythrocyte sedimentation rate (ESR) of $119 \mathrm{~mm} / \mathrm{h}$ the right subclavian artery $(\mathbf{b})$; the aneurysmal dilatation of abdominal aorta, and atrophied left kidney and adrenal gland (c); a pseudoaneurysm of $2.35 \mathrm{~cm} \times 1.74 \mathrm{~cm}$ in the right thoracoacromial artery $(\mathbf{d})$

(reference range $0-15 \mathrm{~mm} / \mathrm{h}$ ) and C-reactive protein (CRP) of $70 \mathrm{mg} / \mathrm{L}$ (reference range 0-8 mg/L). Serum immunoglobulin electrophoresis showed that immunoglobulin G (IgG) and immunoglobulin M (IgM) were elevated. Antinuclear antibody and other autoantibodies, such as antineutrophil cytoplasmic antibody and anticardiolipin antibody, were all negative. A thorough checkup of the vascular system of the whole body did not reveal any new significant 


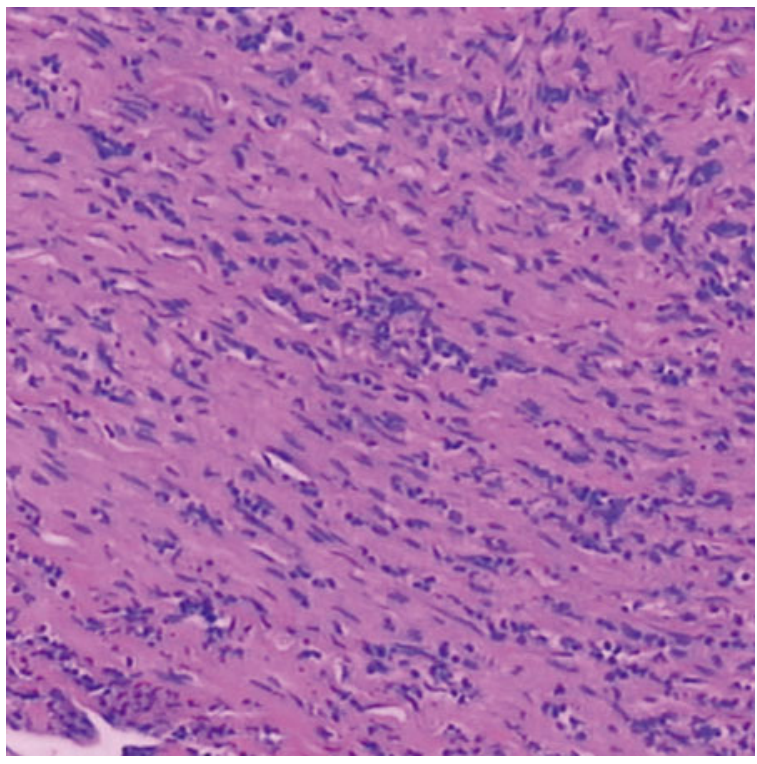

Fig. 2 Diffuse inflammatory cell infiltration in the arterial wall (original magnification $\times 100$ )

abnormality except for a pseudoaneurysm in the right thoracoacromial artery. A diagnosis of vasculo-Behcet's disease was made based on recurrent oral and genital aphthous ulcers, pseudofolliculitis, pseudoaneurysms, and pathergy test. As the disease was active, as evidenced by high ESR, CRP, IgG, IgM, positive pathergy test, and development of fresh aneurysm, the patient was initiated on immunosuppressive therapy using oral prednisone $45 \mathrm{mg} \quad(1 \mathrm{mg} / \mathrm{kg})$ daily and intravenous pulse cyclophosphamide $1,200 \mathrm{mg}$ (12 mg/kg/day for 2 days) every 2 weeks. A month later, the pseudoaneurysm in the right thoracoacromial artery was hardened and the CT angiography revealed that the pseudoaneurysm was shrinking. The levels of ESR, CRP, IgG, and IgM were gradually reduced to the normal range. The patient was discharged on a regimen of azathioprine $100 \mathrm{mg}(2 \mathrm{mg} / \mathrm{kg})$ daily and prednisone $45 \mathrm{mg}$ daily. The dosages of prednisone and azathioprine were tapered gradually. After 16 months, prednisone was reduced to $10 \mathrm{mg}$ every other day. This dose of

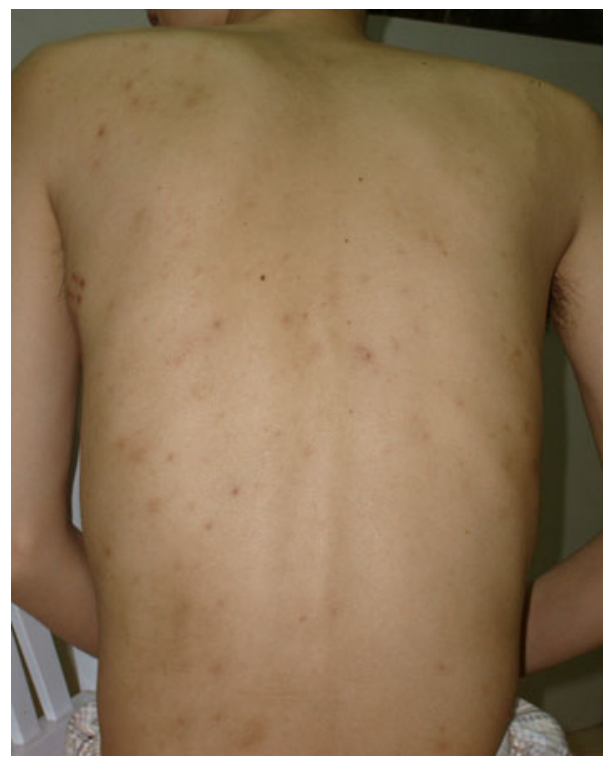

Fig. 3 Multiple pigmented macules on the neck and back prednisone was then continued. After 20 months, azathioprine was reduced to $50 \mathrm{mg} /$ day and discontinued after 30 months. To date, the pseudoaneurysm has regressed without recurrence for approximately 2 years and the patient continues to take prednisone $10 \mathrm{mg}$ every other day.

\section{DISCUSSION}

Behcet's disease with predominant vascular involvement is known as Vasculo-Behcet's disease. It occurs late in the course of Behcet's disease and affects males more frequently than females. Other features of Behcet's disease, such as oral and genital ulcerations, skin lesions, and eye involvement, may not be significant in this subset of patient, as in the present patient [3]. Four different vascular complications have been described: arterial occlusion, arterial aneurysm or pseudoaneurysm, venous thrombosis, and variceal formation [1]. The aorta is the most susceptible site for aneurysm, followed by the pulmonary, femoral, subclavian, popliteal, and carotid arteries [5]. 
It is very important to identify patients with vasculo-Behcet's disease because they are at risk of developing recurrent vascular lesions after the first episode of vascular injury and are prone to progressive multifocal vessel-related complications, as in the present patient [6]. Aneurysms are significantly associated with poor outcome and mainly present acutely with catastrophic hemorrhage. However, there are no specific pathognomonic laboratory tests or histological findings; the diagnosis is based on typical clinical features and natural history [7].

The present case satisfied the international criteria for Behcet's disease [7]. In this case, though the histological finding showed nonspecific arteritis, the history of mucosal ulcerations, cutaneous manifestation, and positive pathergy test distinguished vasculoBehcet's disease from other vasculitides with large artery involvement, including Takayasu's arteritis, giant cell arteritis, Cogan's syndrome, and syphilitic arteritis.

Vasculo-Behcet's disease is a systemic autoimmune inflammatory disorder characterized by vasculitis. Therefore, the aim of treatment is to control vasculitis and regulate the immune system. Various therapeutic modalities have been employed for Behcet's disease, but the optimal medical treatment has not been established, although it predominantly consists of anti-inflammatory and immunosuppressive agents [1]. The present patient was treated with prednisone in combination with cyclophosphamide and azathioprine to control active inflammation. Through this therapy, the levels of ESR, CRP, IgG, and IgM were reduced to the normal range and the pseudoaneurysm in the right thoracoacromial artery regressed. Furthermore, to date, there has not been any new aneurysm or pseudoaneurysm formation. Recently, some reports have shown that infliximab is effective in inducing and maintaining remission of vasculitic activity in patients with Behcet's disease [8]. Infliximab is also effective in patients who do not respond to treatment with corticosteroids and cyclophosphamide [9]. Therefore, in addition to corticosteroids and cyclophosphamide, infliximab should be considered as a good choice in severe vasculo-Behcet's disease.

Surgical and endovascular interventions are also important because of the high risk of aneurismal rupture. Surgical and endovascular interventions alone for aneurysms are usually unsuccessful because the incidence of postoperative complications, such as anastomotic pseudoaneurysm or stent-graft thrombosis, is high, as in the present patient [6]. Some reports have described that surgical complication is less frequent under the remission phase of vasculo-Behcet's disease than under the active phase [10]. Consequently, surgical and endovascular treatment is performed ideally in the clinically inactive period of the disease, and immunosuppressive treatment after surgery helps prevent further vasculopathy and improve the prognosis.

\section{CONCLUSION}

In conclusion, an increased awareness of vasculo-Behcet's disease and its vascular complications is essential. Early diagnosis and early initiation of prednisone in combination with immunosuppressive therapy are critical for inhibiting the progression of vascular lesions and provide a good prognosis.

\section{ACKNOWLEDGMENTS}

No funding or sponsorship was received for this study or publication of this article. Kun Huang 
is the guarantor for this article and takes responsibility for the integrity of the work as a whole.

Conflict of interest. Shuang Li, Ai-Jun Chen, Kun Huang, and Hui Li declare no conflict of interest.

Compliance with Ethics Guidelines. All procedures followed were in accordance with the ethical standards of the responsible committee on human experimentation (institutional and national) and with the Helsinki Declaration of 1975, revised in 2000. Informed consent was obtained from the patient included in this study.

Open Access. This article is distributed under the terms of the Creative Commons Attribution Noncommercial License which permits any noncommercial use, distribution, and reproduction in any medium, provided the original author(s) and the source are credited.

\section{REFERENCES}

1. Mendes D, Correia M, Barbedo M, et al. Behçet's disease-a contemporary review. J Autoimmun. 2009;32:178-88.

2. Alpaqut U, Uqurlucan M, Dayioqlu E. Major arterial involvement and review of Behçet's disease. Ann Vasc Surg. 2007;21:232-9.
3. Fei Y, Li X, Lin S, et al. Major vascular involvement in Behcet's disease: a retrospective study of 796 patients. Clin Rheumatol. 2013 (Epub ahead of print).

4. Sarica-Kucukoqlu R, Akdag-Kose A, Kayaball M, et al. Vascular involvement in Behcet's disease: a retrospective analysis of 2319 cases. Int J Dermatol. 2006;45:919-21.

5. Chae EJ, Do KH, Seo JB, et al. Radiologic and clinical findings of Behcet disease: comprehensive review of multisystemic involvement. Radiographics. 2008;28:e31.

6. Tuzun H, Seyahi E, Arslan C, Hamuryudan V, Besicli $\mathrm{K}$, Yazici H. Management and prognosis of nonpulmonary large arterial disease in patients with Behcet disease. J Vasc Surg. 2012;55:157-63.

7. International Team for the Revision of the International Criteria for Behcet's Disease (ITRICBD); Davatchi F, Assaad-Khalil S, et al. The International Criteria for Behcet's disease (ICBD): a collaborative study of 27 countries on the sensitivity and specificity of the new criteria. J Eur Acad Dermatol Venereol. 2013 (Epub ahead of print).

8. Adler S, Baumgartner I, Villiger PM. Behcet's disease: successful treatment with infliximab in 7 patients with severe vascular manifestations. A retrospective analysis. Arthritis Care Res (Hoboken). 2012;64:607-11.

9. Schreiber BE, Noor N, Juli CF, Haskard DO. Resolution of Behcet's syndrome associated pulmonary arterial aneurysms with infliximab. Semin Arthritis Rheum. 2011;41:482-7.

10. Kalko Y, Basaran M, Aydin U, Kafa U, Basaranoglu $\mathrm{G}$, Yasar T. The surgical treatment of arterial aneurysms in Behçet disease: a report of 16 patients. J Vasc Surg. 2005;42:673-7. 